\title{
Fundamental Solutions of the Instationary Schrödinger Difference Operator
}

\author{
Paula Cerejeiras Nelson Vieira \\ Department of Mathematics, \\ University of Aveiro, \\ 3810-193 Aveiro, Portugal. \\ E-mails: pceres@ua.pt, nvieira@ua.pt
}

October 22, 2018

\begin{abstract}
In this paper we will study the existence of fundamental solutions for the explicit and implicit backward time dependent Schödinger equation, via discrete Fourier transform and its symbol for the Laplace operator. In both cases we will prove that the discrete fundamental solutions obtained converges to the continuous fundamental solution in the $l_{1}$-norm sense.
\end{abstract}

Keywords: Time Dependent Operators, Clifford Analysis, Schrödinger Opeartor, Fundamental Solutions, Difference Operators.

MSC2000: Primary: 30G35; Secondary: 39A12, 65M06

\section{Introduction}

The potential theory is a useful tool to solve boundary value problems by the help of integral equations on the boundary. For constructive analytical considerations and also in the case of numerical applications it is necessary to have an explicit expression of the fundamental solution. However, the integral representations obtained using potential theory are not suitable for an explicit computation of the solutions, due to unacceptable convergence rates of the integral's numerical approximations (for more details see [7]).

A more acceptable alternative in computational terms is given by the use of finite difference approximations. In fact, the connection between the potential theory and finite difference theory is based in the possibility of obtain explicit expressions for the fundamental solutions. This second approach has been applied to elliptic operators, for example, in [3] and [8], where elliptic difference operators were studied and representations for their fundamental solutions were given. However, this approach is restricted to the stationary case. In fact, when 
one takes the time evolution into account, for example, when the operators are parabolic, additional difficulties appear. One of the main difficulties lyes in its fundamental solutions, which must be study in distributional sense.

This paper is based on the work developed in [7, where an orthogonal decomposition of the function spaces in terms of subspaces of null-solutions of the correspondent Dirac operator were given. In order to extend this approach to the nonstationary case we introduce Witt basis, as done in [1. This will gives us the possibility of apply techniques of the elliptic function theory to parabolic domains.

For the study of the continuous Schrödinger equation

$$
\left(-\Delta_{x}-i \partial_{t}\right) u(x, t)=\delta(x, t),
$$

we need to study the fundamental solution of the difference equation

$$
\left(\left(-\Delta_{h}-i \partial_{\tau}\right) E_{h, \tau}\right)(h \underline{m}, \tau k)=\delta_{h, \tau}(h \underline{m}, \tau k) .
$$

For that purpose, two approaches are available to us: either one considers the explicit equation

$$
\left(\left(-\Delta_{h}-i \partial_{\tau}\right) E_{h, \tau}\right)(h \underline{m}, \tau k)=\left\{\begin{array}{ccc}
\frac{1}{h^{3} \tau} & \text { if } & (h \underline{m}, \tau k)=(\underline{0}, 0) \\
0 & \text { if } & (h \underline{m}, \tau k) \neq(\underline{0}, 0)
\end{array},\right.
$$

where $\Delta_{h}, \partial_{\tau}$ and $\delta_{h, \tau}$ are the discrete operators of the Laplacian, the discrete time derivation and the discrete delta function; or we consider the implicit version of the equation (2), with an additional time-step.

The paper will be divided as follows: on Section 2, we present some basic notions about Clifford analysis and finite diferences approximations.

On Section 3 we shall study the existence and behavior of fundamental solutions for the explicit non-stationary Schrödinger equation (2); this study will be divided in three steps: first, we shall prove convergence of the discrete fundamental solution to the continuous one on a bounded domain. On the second step, we prove convergence on a domain of type $G_{h} \times\left[T_{0},+\infty\left[, T_{0}>0\right.\right.$. We will finalize this section with the proof of convergence for the domain $\left.\mathbb{R}_{h}^{3} \times\right] 0,+\infty\left[{ }_{\tau}\right.$.

On Section 4 we prove the existence of a discrete fundamental solution for the implicit Schrödinger equation and that this fundamental solution converges to the continuous one.

In Sections 3 and 4 we will omit a general discussion in discrete spaces of distributions because, on the one hand we want to underline the analogy with the continuous case. On the other hand we are interested in convergence results in norms as strong as possible. Therefore, we investigate if $E_{h, \tau}$ belongs to the space $l_{1}\left(\mathbb{R}_{h}^{3} \times \mathbb{R}_{\tau}^{+}\right)$.

We will present an explicit expression for discrete fundamental solution of both the explicit and implicit backward Schrödinger equation and we will prove that these solutions belongs to the space $l_{1}^{\text {loc }}\left(\mathbb{R}_{h}^{3} \times \mathbb{R}_{\tau}^{+}\right)$. Also, we will prove that these fundamental solutions converges to the continuous one in $l_{1}-$ norm, when the size of the mesh tends to zero. 


\section{Preliminaries}

\subsection{Clifford Analysis}

Consider the $n$-dimensional vector space $\mathbb{R}^{n}$ endowed with a standard orthonormal basis $\left\{e_{1}, \cdots, e_{n}\right\}$ and satisfying the multiplication rules $e_{i} e_{j}+e_{j} e_{i}=$ $-2 \delta_{i, j}$.

We define the universal Clifford algebra $C \ell_{0, n}$ as the $2^{n}$-dimensional associative algebra with basis given by $e_{\emptyset}=1$, as the scalar unit, and $e_{A}=e_{h_{1}} \cdots e_{h_{k}}$, where $A=\left\{h_{1}, \ldots, h_{k}\right\} \subset N=\{1, \ldots, n\}$, for $1 \leq h_{1}<\cdots<h_{k} \leq n$. Each element $x \in C \ell_{0, n}$ will be represented by $x=\sum_{A} x_{A} e_{A}$ and each non-zero vector has their multiplicative inverse given by $\frac{-x}{|x|^{2}}$. We denote the (Clifford) conjugation $x \rightarrow \bar{x}^{C \ell_{0, n}}$ by means of its action on the basis elements

$$
\overline{1}^{C \ell_{0, n}}=1 \quad{\overline{e_{j}}}^{C \ell_{0, n}}=-e_{j} \quad \overline{a b}^{C \ell_{0, n}}=\bar{b}^{C \ell_{0, n}} \bar{a}^{C \ell_{0, n}} .
$$

We introduce the complexified Clifford algebra $C \ell_{n}$ as the tensorial product

$$
\mathbb{C} \otimes C \ell_{0, n}=\left\{w=\sum_{A} z_{A} e_{A}, z_{A} \in \mathbb{C}, A \subset N\right\},
$$

where the imaginary unit interact with the basis elements as $i e_{j}=e_{j} i, j=$ $1, \ldots, n$.

Finally we introduce the conjugate of $w=\sum_{A} z_{A} e_{A}$ as $\bar{w}=\sum_{A}{\overline{z_{A}}}^{C} \overline{e_{A}} C \ell_{0, n}$.

Let now $\Omega \subset \mathbb{R}^{n} \times \mathbb{R}^{+}$denote a bounded domain with sufficiently smooth boundary $\Gamma=\partial \Omega$. A function $u: \Omega \rightarrow C \ell_{n}$ has a representation $u=\sum_{A} u_{A} e_{A}$ with $\mathbb{C}$-valued components $u_{A}$. Properties such as continuity will be understood component-wisely. In the following we will use the short notation $L_{p}(\Omega), C^{k}(\Omega)$, etc., instead of $L_{p}\left(\Omega, C \ell_{n}\right), C^{k}\left(\Omega, C \ell_{n}\right)$. For more details see [4].

We consider the Dirac operator $D=\sum_{j=1}^{n} e_{j} \frac{\partial}{\partial x_{i}}$ which has the property of factorizing the $n$-dimensional Laplacian, that is, $D^{2} u=-\Delta u$. A $C \ell_{n}$-valued function $u$ defined on an open domain $G$, is said to be left-monogenic if it satisfies $D u=0$ on $G$.

Taking into account [1], we will imbed $\mathbb{R}^{n}$ into $\mathbb{R}^{n+2}$. For that purpose we add two new basis elements $\mathfrak{f}$ and $\mathfrak{f}^{\dagger}$ satisfying

$$
\mathfrak{f}^{2}=\mathfrak{f}^{\dagger^{2}}=0, \quad \mathfrak{f f} f^{\dagger}+\mathfrak{f}^{\dagger} \mathfrak{f}=1, \quad \mathfrak{f} e_{j}+e_{j} \mathfrak{f}=\mathfrak{f}^{\dagger} e_{j}+e_{j} \mathfrak{f}^{\dagger}=0, j=1, \cdots, n .
$$

The set $\left\{\mathfrak{f}, \mathfrak{f}^{\dagger}\right\}$ is said to be a Witt basis for $\mathbb{R}^{2}$ and it will allows us to create a suitable factorization of the Schrödinger operator where only partial derivatives are used.

\section{$2.2 \quad$ Finite Difference Calculus}

For each $\emptyset \neq G \subset \mathbb{R}^{n},(n \geq 1)$, we denote the associated discrete domain $G_{h}$, for a fixed mesh size $h>0$, as

$$
G_{h}=\left\{h \underline{m}=\left(h m_{1}, \ldots, h m_{n}\right) \in G: \underline{m} \in \mathbb{Z}^{n}\right\} .
$$


For a continuous function $f$ on $G$, we denote by $R_{h} f$ its restriction to the lattice $G_{h}$ of mesh size $h>0$.

In the following we consider functions restricted to the lattices $\mathbb{R}_{h}^{3}, \mathbb{R}_{\tau}^{+}$. We define the corresponding discrete $l_{1}$-spaces in the usual way:

$$
\begin{aligned}
& u \in l_{1}\left(\mathbb{R}_{h}^{3}\right) \quad \Leftrightarrow\|u\|_{l_{1}\left(\mathbb{R}_{h}^{3}\right)}=\sum_{\underline{m} \in \mathbb{Z}^{3}}|u(h \underline{m})| h^{3}<\infty \\
& v \in l_{1}\left(\mathbb{R}_{\tau}^{+}\right) \quad \Leftrightarrow\|v\|_{l_{1}\left(\mathbb{R}_{\tau}^{+}\right)}=\sum_{k \in \mathbb{N}}|v(\tau k)| \tau \quad<\infty .
\end{aligned}
$$

For a discrete function $u: \mathbb{R}_{h}^{3} \times \mathbb{R}_{\tau}^{+} \rightarrow \mathbb{C}^{4} \sim \mathbb{C} \otimes \mathbb{H}$, given as $u(h \underline{m}, k \tau)=$ $\left(u^{0}, u^{1}, u^{2}, u^{3}\right)$, we have the finite difference approximation for the stationary Dirac operators given by

$$
\begin{aligned}
& D_{h}^{-+} u=\left(\begin{array}{c}
-\partial_{h}^{-1} u^{1}-\partial_{h}^{-2} u^{2}-\partial_{h}^{-3} u^{3} \\
\partial_{h}^{-1} u^{0}-\partial_{h}^{3} u^{2}+\partial_{h}^{2} u^{3} \\
\partial_{h}^{-2} u^{0}+\partial_{h}^{3} u^{1}-\partial_{h}^{1} u^{3} \\
\partial_{h}^{-3} u^{0}-\partial_{h}^{2} u^{1}+\partial_{h}^{1} u^{2}
\end{array}\right), \quad D_{h}^{+-} u=\left(\begin{array}{c}
-\partial_{h}^{1} u^{1}-\partial_{h}^{2} u^{2}-\partial_{h}^{3} u^{3} \\
\partial_{h}^{1} u^{0}-\partial_{h}^{-3} u^{2}+\partial_{h}^{-2} u^{3} \\
\partial_{h}^{2} u^{0}+\partial_{h}^{-3} u^{1}-\partial_{h}^{-1} u^{3} \\
\partial_{h}^{3} u^{0}-\partial_{h}^{-2} u^{1}+\partial_{h}^{-1} u^{2}
\end{array}\right), \\
& u D_{h}^{-+}=\left(\begin{array}{c}
-\partial_{h}^{-1} u^{1}-\partial_{h}^{-2} u^{2}-\partial_{h}^{-3} u^{3} \\
\partial_{h}^{-1} u^{0}+\partial_{h}^{3} u^{2}-\partial_{h}^{2} u^{3} \\
\partial_{h}^{-2} u^{0}-\partial_{h}^{3} u^{1}+\partial_{h}^{1} u^{3} \\
\partial_{h}^{-3} u^{0}+\partial_{h}^{2} u^{1}-\partial_{h}^{1} u^{2}
\end{array}\right), \quad u D_{h}^{+-}=\left(\begin{array}{c}
-\partial_{h}^{1} u^{1}-\partial_{h}^{2} u^{2}-\partial_{h}^{3} u^{3} \\
\partial_{h}^{1} u^{0}+\partial_{h}^{-3} u^{2}-\partial_{h}^{-2} u^{3} \\
\partial_{h}^{2} u^{0}-\partial_{h}^{-3} u^{1}+\partial_{h}^{-1} u^{3} \\
\partial_{h}^{3} u^{0}+\partial_{h}^{-2} u^{1}-\partial_{h}^{-1} u^{2}
\end{array}\right),
\end{aligned}
$$

where

$$
\partial_{h}^{ \pm s} u^{j}=\frac{\left(u^{j}\left(h \underline{m} \pm h e_{s}, k \tau\right)-u^{j}(h \underline{m}, k \tau)\right)}{h}, j=0,1,2,3, s=1,2,3,
$$

represent the spatial forward/backward difference operators. We remark that these difference operators factorize the discrete Laplacian, in the sense that

$$
D_{h}^{+-} D_{h}^{-+} u=D_{h}^{-+} D_{h}^{+-} u=-\Delta_{h} u I_{4}=\left(\sum_{s=1}^{3} \partial_{h}^{-s} \partial_{h}^{s} u^{s}\right) I_{4},
$$

where $I_{4}$ is the $4 \times 4$ identity matrix. In what follows we denote $D_{h}^{+-}$and $D_{h}^{-+}$ as forward/backward difference Dirac operators.

We also have the following (forward) time difference operator (for more details see [7] and [5])

$$
\partial_{\tau} u^{j}(h \underline{m}, k \tau)=\frac{u^{j}(h \underline{m}, \tau(k+1))-u^{j}(h \underline{m}, \tau k)}{\tau}, j=0, \cdots, 3 .
$$

Further, we use the notations

$$
\delta_{h}(h \underline{m})=\left\{\begin{array}{ccc}
\frac{1}{h^{3}} & \text { if } & \underline{m}=(0,0,0) \\
0 & \text { if } & \underline{m} \neq(0,0,0)
\end{array} \quad \text { and } \quad \delta_{\tau}(\tau k)=\left\{\begin{array}{ccc}
\frac{1}{\tau} & \text { if } & k=0 \\
0 & \text { if } & k \neq 0
\end{array}\right.\right.
$$

for the discrete Delta function. 
We will consider the discrete Fourier transform introduced by Stummel (see [7. for more details) with respect to $x$,

$$
\left(\mathcal{F}_{h} u\right)(\xi, \cdot)=\left\{\begin{array}{cl}
\frac{h^{3}}{(2 \pi)^{\frac{3}{2}}} \sum_{h \underline{m} \in \mathbb{Z}^{3}} u(h \underline{m}, \cdot) \exp (i h \underline{m} \xi) & \text { for } \xi \in Q_{h} \\
0 & \text { otherwise }
\end{array},\right.
$$

where $Q_{h}=\left\{\xi=\left(\xi_{1}, \xi_{2}, \xi_{3}\right) \in \mathbb{R}^{3}:-\frac{\pi}{h}<\xi_{1}, \xi_{2}, \xi_{3}<+\frac{\pi}{h}\right\}$.

\section{$3 \quad$ Explicit difference equation}

As it was indicated before, in this section we study the explicit equation (2). This will be done in two steps. In the first one we study the existence of a discrete symbol of the operator in (2), which we use to construct $E_{h, \tau}$, a fundamental solution for discrete explicit Schrödinger backward time-dependent operator. In the second part we will estimate the norm

$$
\left\|E_{h, \tau}-R_{\tau} R_{h} E\right\|_{l_{1}\left(G_{h} \times \mathbb{R}_{\tau}^{+}\right)},
$$

which will allows to calculate its limit when $h$ and $\tau$ tend to zero. Here, $E$ is a fundamental solution for the continuous Schrödinger backward time-dependent operator, where $R_{\tau} R_{h} E$ its restriction to the lattice.

\subsection{Discrete Symbol of the Fundamental Solution}

Let us consider the equation (2). In order to simplify the resolution of this equation, we need to introduce the abbreviation for the symbol of the discrete Laplace operator

$$
d^{2}=\frac{4}{h^{2}}\left(\sin ^{2}\left(\frac{h \xi_{1}}{2}\right)+\sin ^{2}\left(\frac{h \xi_{2}}{2}\right)+\sin ^{2}\left(\frac{h \xi_{3}}{2}\right)\right) .
$$

Applying the discrete Fourier transform to (2), we get the equation

$$
\left(\left(d^{2} \mathcal{F}_{h} E_{h, \tau}-i \partial_{\tau} \mathcal{F}_{h} E_{h, \tau}\right)\right)(\xi, t)=\frac{1}{(2 \pi)^{\frac{3}{2}}} \delta_{\tau}(t) \chi_{h}(\xi),
$$

with $\chi_{\tau}$ being the characteristic function of $Q_{h}$, which has the solution

$$
\left(\mathcal{F}_{h} E_{h, \tau}\right)(\xi, t)=\frac{i}{2 \pi} H(t)\left(1-i \tau d^{2}\right)^{\frac{t}{\tau}-1} \chi_{h}(\xi) .
$$

Using the restriction of the continuous inverse Fourier transform $\mathcal{F}$, to the $\mathbb{R}_{h}^{3}, \mathcal{F}_{h}^{-1}=R_{h} \mathcal{F}$, which acts as an inverse for $\mathcal{F}_{h}$, we obtain

$$
E_{h, \tau}(h \underline{m}, \tau k)=i H(\tau k)\left(\left(1-i \tau \Delta_{h}\right)^{k-1} \delta_{h}\right)(h \underline{m}),
$$

which is a fundamental solution of the discrete explicit Schrödinger backward time-dependent equation. 


\subsection{Convergence Result}

As it was indicated before, we will now estimate the norm

$$
\left\|E_{h, \tau}-R_{\tau} R_{h} E\right\|_{l_{1}\left(G_{h} \times \mathbb{R}_{\tau}^{+}\right)} .
$$

This estimation will be done in two parts. In the first we will consider the case of the time limited interval, i.e., we will estimate our norm in $\left.\left.G_{h} \times\right] 0, T_{0}\right]_{\tau}$, with $T_{0} \in \mathbb{R}^{+}$. In the second part we will consider our norm in the $\left.\left.G_{h} \times\right] T_{0},+\infty\right]$.

\subsubsection{Case of the Limited Time Interval}

Initially we shall study now the behavior of

$$
\left\|E_{h, \tau}-R_{\tau} R_{h} E\right\|_{\left.\left.l_{1}\left(G_{h} \times\right] 0, T_{0}\right]_{\tau}\right)} .
$$

For this purpose we rewrite the equation (2) in the form

$$
\begin{aligned}
E_{h, \tau}(h \underline{\underline{m}}, \tau(k+1)) \\
\quad=\left(1+\frac{6 i \tau}{h^{2}}\right) E_{h, \tau}(h \underline{m}, \tau k) \\
\quad+\frac{i \tau}{h^{2}}\left[E_{h, \tau}\left(h\left(m_{1}+1\right), h m_{2}, h m_{3}, \tau k\right)+E_{h, \tau}\left(h\left(m_{1}-1\right), h m_{2}, h m_{3}, \tau k\right)\right. \\
\quad+E_{h, \tau}\left(h m_{1}, h\left(m_{2}+1\right), h m_{3}, \tau k\right)+E_{h, \tau}\left(h m_{1}, h\left(m_{2}-1\right), h m_{3}, \tau k\right) \\
\left.\quad+E_{h, \tau}\left(h m_{1}, h m_{2}, h\left(m_{3}+1\right), \tau k\right)+E_{h, \tau}\left(h m_{1}, h m_{2}, h\left(m_{3}-1\right), \tau k\right)\right]
\end{aligned}
$$

which implies that $E_{h, \tau}$ is supported in a cone. From (5) and (6) we get

$$
\left\|E_{h, \tau}(\cdot, \tau)\right\|_{l_{1}\left(\mathbb{R}_{h}^{3}\right)} \leq 1 \quad\left\|E_{h, \tau}(\cdot, \tau k)\right\|_{l_{1}\left(\mathbb{R}_{h}^{3}\right)} \leq 1 \quad\left\|E_{h, \tau}(\cdot, \tau(k+1))\right\|_{l_{1}\left(\mathbb{R}_{h}^{3}\right)} \leq 1 .
$$

Further, let $T_{0}=\tau m_{0}$, with $m_{0} \in \mathbb{N}$ and $T_{0} \in \mathbb{R}^{+}$. By addition with respect to $t$, we get the estimation

$$
\left\|E_{h, \tau}\right\|_{\left.\left.l_{1}\left(G_{h} \times\right] 0, T_{0}\right]_{\tau}\right)} \leq\left\|E_{h, \tau}\right\|_{\left.\left.l_{1}\left(\mathbb{R}_{h}^{3} \times\right] 0, T_{0}\right]_{\tau}\right)} \leq \sum_{k=1}^{m_{0}} \tau=T_{0} .
$$

Now we consider the continuous fundamental solution (see [2] for more details)

$$
E(x, t)=\frac{i H(t)}{(4 i \pi t)^{\frac{3}{2}}} \exp \left(\frac{i|x|^{2}}{4 t}\right)
$$

of the continuous Schrödinger backward time-dependent operator. 
We get

$$
\begin{aligned}
\left\|R_{h} E(\cdot, t)\right\|_{l_{1}\left(G_{h}\right) \leq} \leq & H(t) \sum_{h \underline{m} \in G_{h}}\left|\frac{i}{(4 i \pi t)^{\frac{3}{2}}}\right|\left|\exp \left(\frac{i h^{2}|\underline{m}|^{2}}{4 t}\right)\right| h^{3} \\
= & \frac{H(t)}{(4 \pi t)^{\frac{3}{2}}} \sum_{h \underline{m} \in G_{h}} h^{3} \\
& \frac{H(t)}{(4 \pi t)^{\frac{3}{2}}} \operatorname{Vol}\left(G_{h}\right),
\end{aligned}
$$

where $\operatorname{Vol}\left(G_{h}\right)=\sum_{h \underline{m} \in G_{h}} h^{3}$. Furthermore

$$
\begin{aligned}
\left\|R_{\tau} R_{h} E\right\|_{\left.\left.l_{1}\left(G_{h} \times\right] 0, T_{0}\right]_{\tau}\right)} & =\sum_{k=1}^{m_{0}} \frac{\operatorname{Vol}\left(G_{h}\right)}{(4 \pi \tau k)^{\frac{3}{2}}} \tau \\
& =\frac{\operatorname{Vol}\left(G_{h}\right)}{(4 \pi \tau)^{\frac{3}{2}} \tau^{\frac{1}{2}}} \sum_{k=1}^{m_{0}} \frac{1}{k^{\frac{3}{2}}} \\
& \leq\left\{\begin{array}{ccc}
\operatorname{Vol}\left(G_{h}\right) \frac{T_{0}}{(4 \pi)^{\frac{3}{2}}} & \text { if } & \tau \geq 1 \\
\operatorname{Vol}\left(G_{h}\right) \frac{T_{0}+m_{0}^{2}}{(4 \pi)^{\frac{3}{2}}} & \text { if } & 0<\tau<1
\end{array} .\right.
\end{aligned}
$$

From (6) and (8) we conclude

$$
\left\|E_{h, \tau}-R_{\tau} R_{h} E\right\|_{\left.\left.l_{1}\left(G_{h} \times\right] 0, T_{0}\right]_{\tau}\right)} \leq\left\{\begin{array}{ccc}
T_{0}+\operatorname{Vol}\left(G_{h}\right) \frac{T_{0}}{(4 \pi)^{\frac{3}{2}}} & \text { if } & \tau \geq 1 \\
T_{0}+\operatorname{Vol}\left(G_{h}\right) \frac{T_{0}+m_{0}^{2}}{(4 \pi)^{\frac{3}{2}}} & \text { if } & 0<\tau<1
\end{array}\right.
$$

The previous inequality describes the approximation error of the fundamental solution (5) for small values of time variable.

\subsubsection{Case of the Unlimited Time Interval}

In the following, for $t=\tau k \in \mathbb{R}_{\tau}^{+}$with $t>T_{0}$, we study

$$
\left\|E_{h, \tau}(\cdot, t)-R_{\tau} R_{h} E(\cdot, t)\right\|_{l_{1}\left(G_{h}\right)}
$$

In order to guarantee the convergence of some series and integrals, we need to consider the following regularized fundamental solution of the Schrödinger operator

$$
E^{\epsilon}(x, t)=\frac{i H(t)}{(4 i \pi t)^{\frac{3}{2}}} \exp \left(\frac{(-\epsilon+i)|x|^{2}}{4 t}\right)
$$


which converges, in $L_{p}\left(G \times \mathbb{R}^{+}\right)$, with $1 \leq p<+\infty$, to the continuous fundamental solution (7).

We have

$$
\begin{aligned}
& \| E_{h, \tau}(\cdot, \tau k)-\left.R_{\tau} R_{h} E(\cdot, \tau k)\right|_{l_{1}\left(G_{h}\right)} \\
& =\sum_{h \underline{m} \in G_{h}}\left|E_{h, \tau}(h \underline{m}, \tau k)-R_{\tau} R_{h} E(h \underline{m}, \tau k)\right| h^{3} \\
& \leq \operatorname{Vol}\left(G_{h}\right) \max _{h \underline{m} \in G_{h}}\left|E_{h, \tau}(h \underline{m}, \tau k)-R_{\tau} R_{h} E(h \underline{m}, \tau k)\right| \\
& =\operatorname{Vol}\left(G_{h}\right) \max _{h \underline{m} \in G_{h}}\left|\left(R_{h} \mathcal{F} \mathcal{F}_{h} E_{h, \tau}\right)(h \underline{m}, \tau k)-\left(R_{\tau} R_{h} \mathcal{F} \mathcal{F}^{-1} E\right)(h \underline{m}, \tau k)\right| \\
& =\operatorname{Vol}\left(G_{h}\right) \max _{h \underline{m} \in G_{h}}\left[\left|\left(R_{h} \mathcal{F} \mathcal{F}_{h} E_{h, \tau}\right)(h \underline{m}, \tau k)-\left(R_{\tau} R_{h} \mathcal{F} \mathcal{F}^{-1} E^{\epsilon}\right)(h \underline{m}, \tau k)\right|\right. \\
& \left.\quad+\left|\left(R_{\tau} R_{h} \mathcal{F} \mathcal{F}^{-1} E^{\epsilon}\right)(h \underline{m}, \tau k)-\left(R_{\tau} R_{h} \mathcal{F} \mathcal{F}^{-1} E\right)(h \underline{m}, \tau k)\right|\right] \\
& \leq \operatorname{Vol}\left(G_{h}\right)\left[\left|\frac{1}{2 \pi} \int_{\mathbb{R}^{3}}\left[\left(\mathcal{F}_{h} E_{h, \tau}\right)(x, \tau k)-\left(R_{\tau} \mathcal{F}^{-1} E\right)(x, \tau k)\right] \exp (-i x \xi) d \xi\right|\right. \\
& \left.\quad+\max _{h \underline{m} \in G_{h}}\left|\left(R_{\tau} R_{h} \mathcal{F} \mathcal{F}^{-1} E^{\epsilon}\right)(h \underline{m}, \tau k)-\left(R_{\tau} R_{h} \mathcal{F} \mathcal{F}^{-1} E\right)(h \underline{m}, \tau k)\right|\right] \\
& \leq \frac{\operatorname{Vol}\left(G_{h}\right)}{2 \pi}[\underbrace{}_{\mathbb{R}^{3}}\left|\left(\mathcal{F}_{h} E_{h, \tau}\right)(x, \tau k)-\left(R_{\tau} \mathcal{F}^{-1} E\right)(x, \tau k)\right||\exp (-i x \xi)| d \xi \\
& \left.\quad+\max _{h \underline{m} \in G_{h}}\left|\left(R_{\tau} R_{h} \mathcal{F} \mathcal{F}^{-1} E^{\epsilon}\right)(h \underline{m}, \tau k)-\left(R_{\tau} R_{h} \mathcal{F} \mathcal{F}^{-1} E\right)(h \underline{m}, \tau k)\right|\right] \\
& =\frac{\operatorname{Vol}\left(G_{h}\right)}{2 \pi}\left[\begin{array}{c}
\left.\|\left(\mathcal{F}_{h} E_{h, \tau}\right)(\cdot, \tau k)-\left(R_{\tau} \mathcal{F}^{-1} E^{\epsilon}\right)(\cdot, \tau k) \mid \mathcal{F}^{-1} E^{\epsilon}\right)(h \underline{m}, \tau k)-\left(R_{\tau} R_{h} \mathcal{F} \mathcal{F}^{-1} E\right)(h \underline{m}, \tau k) \mid \\
(I)
\end{array} .\right.
\end{aligned}
$$

By the convergence of the regularized fundamental solution $E^{\epsilon}$ to the continuous one $E$, we conclude immediately that the term $(I I)$ converges to zero, as $\epsilon$ goes to $0^{+}$. This fact implies that the study of (10) depends on the analysis of the term $(I)$. In this case, we need to split this study into the outside of $Q_{h}$ and its inside. 


$$
\begin{aligned}
& \text { In } \mathbb{R}^{3} \backslash Q_{h}, \text { we have for the term }(I) \\
& \begin{aligned}
\left\|\left(\mathcal{F}_{h} E_{h, \tau}\right)(\cdot, \tau k)-\left(R_{\tau} \mathcal{F}^{-1} E^{\epsilon}\right)(\cdot, \tau k)\right\|_{L_{1}\left(\mathbb{R}^{3}\right)} \\
=\left\|\left(R_{\tau} \mathcal{F}^{-1} E^{\epsilon}\right)(\cdot, \tau k)\right\|_{L_{1}\left(\mathbb{R}^{3}\right)} \\
=\frac{H(\tau k)}{(4 \pi t)^{\frac{3}{2}}} \frac{(2 \tau k)^{3}}{\epsilon^{\frac{3}{2}}}\left\|\exp \left(-\frac{\tau k|\xi|^{2}}{\epsilon}\right) \exp \left(-i\left(\tau k|\xi|^{2}-\frac{\pi}{4}\right)\right)\right\|_{L_{1}\left(\mathbb{R}^{3} \backslash Q_{h}\right)} \\
=\frac{H(\tau k) 2 \epsilon^{\frac{3}{2}}}{(\pi \tau k)^{\frac{3}{2}}} \exp \left(-\frac{\tau k \pi^{2}}{h^{2} \epsilon}\right) .
\end{aligned}
\end{aligned}
$$

In $Q_{h}$ we have for $(I)$ the estimation

$$
\begin{aligned}
\left\|\left(\mathcal{F}_{h} E_{h, \tau}\right)(\cdot, \tau k)-\left(R_{\tau} \mathcal{F}^{-1} E^{\epsilon}\right)(\cdot, \tau k)\right\|_{L_{1}\left(\mathbb{R}^{3}\right)} & \frac{H(\tau k)}{(2 \pi)^{\frac{3}{2}}}\left\|\left(1-i \tau d^{2}\right)^{k}-\left(\frac{2 \tau k}{\epsilon}\right)^{\frac{3}{2}} \exp \left(-\frac{\tau k|\xi|^{2}}{\epsilon}\right) \exp \left(-i\left(\tau k|\xi|^{2}-\frac{3 \pi}{4}\right)\right)\right\|_{L_{1}\left(\mathbb{R}^{3}\right)} \\
= & \frac{H(\tau k)}{(2 \pi)^{\frac{3}{2}}}\left(\frac{2 \tau k}{\epsilon}\right)^{\frac{3}{2}} \\
& \cdot\left\|\left(\frac{\epsilon}{2 \tau k}\right)^{\frac{3}{2}}\left(1-i \tau d^{2}\right)^{k}-\exp \left(-\frac{\tau k|\xi|^{2}}{\epsilon}\right) \exp \left(-i\left(\tau k|\xi|^{2}-\frac{3 \pi}{4}\right)\right)\right\|_{L_{1}\left(\mathbb{R}^{3}\right)} \\
\leq & \frac{H(\tau k)}{(2 \pi)^{\frac{3}{2}}}\left(\frac{2 \tau k}{\epsilon}\right)^{\frac{3}{2}} \\
& {\left[\begin{array}{l}
\left\|\left(\frac{\epsilon}{2 \tau k}\right)^{\frac{3}{2}}\left(1-i \tau d^{2}\right)^{k}-\exp \left(-\frac{\tau k d^{2}}{\epsilon}\right) \exp \left(-i\left(\tau k d^{2}-\frac{3 \pi}{4}\right)\right)\right\|_{L_{1}\left(\mathbb{R}^{3}\right)} \\
+\| \exp \left(-\frac{\tau k d^{2}}{\epsilon}\right)^{\frac{3}{2}} \exp \left(-i\left(\tau k d^{2}-\frac{3 \pi}{4}\right)\right) \\
-\exp \left(-\frac{\tau k|\xi|^{2}}{\epsilon}\right)^{\frac{3}{2}} \exp \left(-i\left(\tau k|\xi|^{2}-\frac{3 \pi}{4}\right)\right) \|_{L_{1}\left(\mathbb{R}^{3}\right)}
\end{array}\right.}
\end{aligned}
$$


From

$$
\begin{aligned}
\frac{|\xi|^{2}-d^{2}}{\epsilon} & =\left[|\xi|^{2}-\frac{4}{h^{2}}\left(\sin ^{2}\left(\frac{h \xi_{1}}{2}\right)+\sin ^{2}\left(\frac{h \xi_{2}}{2}\right)+\sin ^{2}\left(\frac{h \xi_{3}}{2}\right)\right)\right] \frac{1}{\epsilon} \\
& \leq\left[\frac{h^{2}}{12}\left(\xi_{1}^{4}+\xi_{2}^{4}+\xi_{3}^{4}\right)\right] \frac{1}{\epsilon} \\
& \leq \frac{h^{2}|\xi|^{2}}{12 \epsilon}
\end{aligned}
$$

we get

$$
\begin{aligned}
& \left|\exp \left(-\frac{\tau k d^{2}}{\epsilon}\right)^{\frac{3}{2}} \exp \left(-i\left(\tau k d^{2}-\frac{3 \pi}{4}\right)\right)-\exp \left(-\frac{\tau k|\xi|^{2}}{\epsilon}\right)^{\frac{3}{2}} \exp \left(-i\left(\tau k|\xi|^{2}-\frac{3 \pi}{4}\right)\right)\right| \\
& \leq\left|\exp \left(-\frac{\tau k d^{2}}{\epsilon}\right)-\exp \left(-\frac{\tau k|\xi|^{2}}{\epsilon}\right)\right|\left|\exp \left(-i\left(\tau k|\xi|^{2}-\frac{3 \pi}{4}\right)\right)\right| \\
& \quad+\left|\exp \left(-\frac{\tau k|\xi|^{2}}{\epsilon}\right)\right|\left|\exp \left(-i\left(\tau k d^{2}-\frac{3 \pi}{4}\right)\right)-\exp \left(-i\left(\tau k|\xi|^{2}-\frac{3 \pi}{4}\right)\right)\right| \\
& \leq\left|\exp \left(-\frac{\tau k d^{2}}{\epsilon}\right)-\exp \left(-\frac{\tau k|\xi|^{2}}{\epsilon}\right)\right|+2 \exp \left(-\frac{\tau k|\xi|^{2}}{\epsilon}\right) \\
& \leq \tau k\left(\frac{|\xi|^{2}-d^{2}}{\epsilon}\right) \exp \left(-\frac{\tau k d^{2}}{\epsilon}\right)+2 \exp \left(-\frac{\tau k|\xi|^{2}}{\epsilon}\right) \\
& \leq \frac{\tau k h^{2}|\xi|^{2}}{12 \epsilon} \exp \left(-\frac{4|\xi|^{2} \tau k}{\pi^{2} \epsilon}\right)+2 \exp \left(-\frac{\tau k|\xi|^{2}}{\epsilon}\right)
\end{aligned}
$$

which implies that $(I V)$ satisfies

$$
\begin{aligned}
(I V) \leq \frac{\tau k h^{2}}{3 \epsilon} \int_{0}^{\frac{\sqrt{2} \pi}{h}} \int_{0}^{\frac{\pi}{2}} \int_{-\frac{\pi}{h}}^{\frac{\pi}{h}} r^{5} \exp \left(-\frac{4 r^{2} \tau k}{\pi^{2} \epsilon}\right) d z d \varphi d r \\
\quad+2 \int_{0}^{\frac{\sqrt{2} \pi}{h}} \int_{0}^{\frac{\pi}{2}} \int_{-\frac{\pi}{h}}^{\frac{\pi}{h}} \exp \left(-\frac{(\tau k)^{2}}{\epsilon}\right) d z d \varphi d r \\
\leq \frac{\pi^{8}}{192}\left[\exp \left(-\frac{8 \tau k}{\epsilon h^{2}}\right)\left(\frac{\epsilon^{2} h}{(\tau k)^{2}}+\frac{8 \epsilon}{\tau k h}+\frac{32}{h^{3}}\right)-\frac{\epsilon^{2} h}{(\tau k)^{2}}\right] \\
+\frac{2 \pi^{2}}{h}\left(\frac{\pi \epsilon}{\tau k\left(h^{2}-1\right)}\right)^{\frac{1}{2}} \int_{0}^{2 \sqrt{\frac{2 \tau k}{\epsilon h}}} \exp \left(-y^{2}\right) d y
\end{aligned}
$$


Also, we have the following relation

$$
\begin{aligned}
& \left|\left(\frac{\epsilon}{2 \tau k}\right)^{\frac{3}{2}}\left(1-i \tau d^{2}\right)^{k}-\exp \left(-\frac{\tau k d^{2}}{\epsilon}\right) \exp \left(-i\left(\tau k d^{2}-\frac{3 \pi}{4}\right)\right)\right| \\
& \quad \leq\left(\frac{\epsilon}{2 \tau k}\right)^{\frac{3}{2}}\left|1-i \tau d^{2}\right|^{k}+\exp \left(-\frac{\tau k d^{2}}{\epsilon}\right) \\
& \quad \leq\left(\frac{\epsilon}{2 \tau k}\right)^{\frac{3}{2}}\left(1+\tau^{2} d^{4}\right)^{k}+\exp \left(-\frac{\tau k d^{2}}{\epsilon}\right) \\
& \quad \leq\left(\frac{\epsilon}{2 \tau k}\right)^{\frac{3}{2}}\left(1+\tau^{2}\left(h^{2}-1\right)^{2}|\xi|^{4}\right)^{k}+\exp \left(-\frac{\tau k\left(h^{2}-1\right)|\xi|^{2}}{\epsilon}\right),
\end{aligned}
$$

which implies that

$$
\begin{aligned}
(I I I) \leq & \left(\frac{\epsilon}{2 \tau k}\right)^{\frac{3}{2}} \int_{0}^{\frac{\sqrt{2} \pi}{h}} \int_{0}^{\frac{\pi}{2}} \int_{-\frac{\pi}{h}}^{\frac{\pi}{h}}\left(1+\tau^{2}\left(h^{2}-1\right)^{2} r^{4}\right)^{k} d z d \varphi d r \\
& +\int_{0}^{\frac{\sqrt{2} \pi}{h}} \int_{0}^{\frac{\pi}{2}} \int_{-\frac{\pi}{h}}^{\frac{\pi}{h}} \exp \left(-\frac{\tau k\left(h^{2}-1\right) r^{2}}{\epsilon}\right) d z d \varphi d r \\
= & \frac{\epsilon \pi^{2}}{\tau k h} \int_{0}^{\frac{\sqrt{2} \pi}{h}}\left(1+\tau^{2}\left(h^{2}-1\right)^{2} r^{4}\right)^{k} d r \\
& +\frac{\pi^{2}}{h} \int_{0}^{\frac{\sqrt{2} \pi}{h}} \exp \left(-\frac{\tau k\left(h^{2}-1\right) r^{2}}{\epsilon}\right) d r \\
= & \frac{\epsilon \pi^{2}}{\tau k h} C_{1}(\epsilon, h, \tau)+\frac{\pi^{2}}{2 h}\left(\frac{\pi \epsilon}{\tau k\left(h^{2}-1\right)}\right)^{\frac{1}{2}} \int_{0}^{\frac{\pi}{h} \sqrt{\frac{2 \tau k\left(h^{2}-1\right)}{\epsilon}}} \exp \left(-y^{2}\right) d y
\end{aligned}
$$

where

$$
C_{1}(\epsilon, h, \tau)=\int_{0}^{\frac{\sqrt{2} \pi}{h}}\left(1+\tau^{2}\left(h^{2}-1\right)^{2} r^{4}\right)^{k} d r
$$

tends to zero when $\epsilon<\tau^{8}$ and $\frac{\tau}{h^{2}}<\frac{1}{6 \pi^{2}}$.

Taking into account the estimates obtained previously for the terms (III) 
and $(I V)$ we conclude that inside $Q_{h}$ we have

$$
\begin{aligned}
& \text { (12) } \leq \frac{H(t)}{(2 \pi)^{\frac{3}{2}}}\left(\frac{2 \tau k}{\epsilon}\right)^{\frac{3}{2}}\left[\frac{\epsilon \pi^{2}}{\tau k h} C_{1}(\epsilon, h, \tau)\right. \\
& +\frac{\pi^{2}}{2 h} \sqrt{\frac{\pi \epsilon}{\tau k\left(h^{2}-1\right)}} \int_{0}^{\frac{\pi}{h} \sqrt{\frac{2 \tau k\left(h^{2}-1\right)}{\epsilon}}} \exp \left(-y^{2}\right) d y \\
& +\frac{\pi^{8}}{192}\left[\exp \left(-\frac{8 \tau k}{\epsilon h^{2}}\right)\left(\frac{\epsilon^{2} h}{(\tau k)^{2}}+\frac{8 \epsilon}{\tau k h}+\frac{32}{h^{3}}\right)-\frac{\epsilon^{2} h}{(\tau k)^{2}}\right] \\
& \left.+\frac{2 \pi^{2}}{h} \sqrt{\frac{\epsilon}{\tau k}} \int_{0}^{\frac{2}{h} \sqrt{\frac{2 \tau k}{\epsilon}}} \exp \left(y^{2}\right) d y,\right] .
\end{aligned}
$$

Taking into account the estimations obtained in (11) and (15), we conclude that

$$
\begin{gathered}
\left\|E_{h, \tau}(\cdot, \tau k)-R_{\tau} R_{h} E(\cdot, \tau k)\right\|_{l_{1}\left(G_{h}\right)} \\
=\frac{V o l\left(G_{h}\right)}{2 \pi}\left[2 H(\tau k)\left(\frac{\epsilon}{\pi \tau k}\right)^{\frac{3}{2}} \exp \left(-\frac{\tau k \pi^{2}}{h^{2} \epsilon}\right)\right. \\
+H(\tau k)\left(\frac{2 \tau k}{2 \pi \epsilon}\right)^{\frac{3}{2}}\left[\frac{\epsilon \pi^{2}}{\tau k h} C_{1}(\epsilon, h, \tau)\right. \\
+\frac{\pi^{2}}{2 h} \sqrt{\frac{\pi \epsilon}{\tau k\left(h^{2}-1\right)}} \int_{0}^{\frac{\pi}{h}} \frac{\sqrt{\frac{2 \tau k\left(h^{2}-1\right)}{\epsilon}}}{\exp \left(-y^{2}\right) d y} \\
+\frac{\pi^{8}}{192}\left[\exp \left(-\frac{8 \tau k}{\epsilon h^{2}}\right)\left(\frac{\epsilon^{2} h}{(\tau k)^{2}}+\frac{8 \epsilon}{\tau k h}+\frac{32}{h^{3}}\right)-\frac{\epsilon^{2} h}{(\tau k)^{2}}\right] \\
\left.\left.+\frac{2 \pi^{2}}{h} \sqrt{\frac{\epsilon}{\tau k}} \int_{0}^{\frac{2}{h} \sqrt{\frac{2 \tau k}{\epsilon}}} \exp \left(y^{2}\right) d y,\right]\right] .
\end{gathered}
$$

Evaluating now the $l_{1}-$ norm with respect to the time-lattice

$$
\begin{aligned}
& \left\|E_{h, \tau}(\cdot, \tau k)-R_{\tau} R_{h} E(\cdot, \tau k)\right\|_{l_{1}\left(G_{h} \times\left(T_{0},+\infty\right)_{\tau}\right)} \\
& =\|\| E_{h, \tau}(\cdot, \tau k)-R_{\tau} R_{h} E(\cdot, \tau k)\left\|_{l_{1}\left(G_{h}\right)}\right\|_{l_{1}\left(\left(T_{0},+\infty\right)_{\tau}\right)} \\
& \quad=\sum_{k=m_{0}+1}^{+\infty} \tau\left\|E_{h, \tau}(\cdot, \tau k)-R_{\tau} R_{h} E(\cdot, \tau k)\right\|_{l_{1}\left(G_{h}\right)}
\end{aligned}
$$




$$
\begin{aligned}
=\frac{\operatorname{Vol}\left(G_{h}\right)}{2 \pi} \sum_{k=m_{0}+1}^{+\infty}[2 & \left(\frac{2 \epsilon}{\pi k \tau}\right)^{\frac{3}{2}} \exp \left(-\frac{k \tau \pi^{2}}{h^{2} \epsilon}\right)+\left(\frac{k \tau}{\pi \epsilon}\right)^{\frac{3}{2}}\left[\frac{\epsilon \pi^{2}}{k \tau h} C_{1}(\epsilon, h, \tau)\right. \\
& +\frac{\pi^{2}}{2 h} \sqrt{\frac{\pi \epsilon}{k \tau\left(h^{2}-1\right)}} \int_{0}^{\frac{\pi}{h} \sqrt{\frac{2 k \tau\left(h^{2}-1\right)}{\epsilon}}} \exp \left(-y^{2}\right) d y \\
& +\frac{\pi^{8}}{192}\left[\exp \left(-\frac{8 k \tau}{\epsilon h^{2}}\right)\left(\frac{\epsilon^{2} h}{(k \tau)^{2}}+\frac{8 \epsilon}{k \tau h}+\frac{32}{h^{3}}\right)-\frac{\epsilon^{2} h}{(k \tau)^{2}}\right] \\
& \left.\left.+\frac{2 \pi^{2}}{h} \sqrt{\frac{\epsilon}{k \tau}} \int_{0}^{\frac{2}{h} \sqrt{\frac{2 \epsilon}{k \tau}}} \exp \left(-y^{2}\right) d y\right]\right]
\end{aligned}
$$

After straightforward calculations we conclude that the previous series is convergent and its sums, which we will denote by $C_{2}(h, h, \tau)$, tends to zero when $\epsilon<\tau^{8}$ and $\frac{\tau}{h^{2}}<\frac{1}{6 \pi^{2}}$.

\subsubsection{Main Result}

Using the inequalities (9) and (17) we obtain the general estimation

$$
\leq\left\{\begin{array}{ccc}
T_{0}+\operatorname{Vol}\left(G_{h}\right) \frac{T_{0}}{(4 \pi)^{\frac{3}{2}}}+C_{2}(\epsilon, h, \tau) & \text { if } & \tau \geq 1 \wedge\left(\epsilon<\tau^{8} \wedge \frac{\tau}{h^{2}}<\frac{1}{6 \pi^{2}}\right) \\
T_{0}+\operatorname{Vol}\left(G_{h}\right) \frac{T_{0}+m_{0}^{2}}{(4 \pi)^{\frac{3}{2}}}+C_{2}(\epsilon, h, \tau) & \text { if } \quad 0<\tau<1 \wedge\left(\epsilon<\tau^{8} \wedge \frac{\tau}{h^{2}}<\frac{1}{6 \pi^{2}}\right)
\end{array} .\right.
$$

For the purpose of our convergence theorem we require that $h \leq h_{0}$, where $h_{0}$ is an arbitrary constant. Now we can formulate the following convergence theorem

Theorem 3.1. Let $\frac{\tau^{2}}{h^{2}}<\frac{1}{6 \pi^{2}}$. Then

$$
\left\|E_{h, \tau}-R_{\tau} R_{h} E\right\|_{l_{1}\left(G_{h} \times \mathbb{R}_{\tau}^{+}\right)} \rightarrow 0 \text { for } h, \tau \rightarrow 0^{+} .
$$

Proof. We prove that for an arbitrary $\delta>0$ there exists a constant $h$ such that

$$
\left\|E_{h, \tau}-R_{\tau} R_{h} E\right\|_{l_{1}\left(G_{h} \times \mathbb{R}_{\tau}^{+}\right)} \leq \delta
$$

Also, it is possible to choose a $T_{0}$ in the lattice $\mathbb{R}_{\tau}^{+}$, and define

$$
T_{0}^{+}=T_{0}+\alpha \tau \quad \text { and } \quad T_{0}^{-}=T_{0}-(1-\alpha) \tau \quad \text { with } \alpha \in[0,1)
$$

such that $T_{0}^{+} \in \mathbb{R}_{\tau}^{+}$and $T_{0}^{-} \in \mathbb{R}_{\tau}^{+}$. Obviously we have

$$
\begin{aligned}
& \left\|E_{h, \tau}-R_{\tau} R_{h} E\right\|_{l_{1}\left(G_{h} \times \mathbb{R}_{\tau}^{+}\right)} \\
& \quad \leq\left\|E_{h, \tau}-R_{\tau} R_{h} E\right\|_{\left.\left.l_{1}\left(G_{h} \times\right] 0, T_{0}^{+}\right]_{\tau}\right)}+\left.\left\|E_{h, \tau}-R_{\tau} R_{h} E\right\|\right|_{l_{1}\left(G_{h} \times\left(T_{0}^{-},+\infty\right)_{\tau}\right)} .
\end{aligned}
$$


Now, a simple estimation using (9) and (17) shows that the right-hand side of the last inequality is bounded by $\delta$.

\section{Implicit Difference Equation}

In this section we will make a similar study for the implicit equation, i.e, we will initially obtain the fundamental solution $E_{h, \tau}^{*}$ of the following implicit equation

$$
\left(-\Delta_{h} E_{h, \tau}^{*}\right)(h \underline{m}, \tau(k+1))-i\left(\partial_{\tau} E_{h, \tau}^{*}\right)(h \underline{m}, \tau k)=\delta_{h}(h \underline{m}) \delta_{\tau}(\tau k),
$$

and then we will develop similar convergent results for this solution.

Using the discrete Fourier transform again we find the solution

$$
\left(\mathcal{F}_{h} E_{h, \tau}^{*}\right)(\xi, t)=\frac{i}{2 \pi}\left(1+i \tau d^{2}\right)^{-\frac{t}{\tau}} \chi_{h}(\xi)
$$

in analogy to (4). Finally, we get the following system of equations to calculate $E_{h, \tau}^{*}(x, t)$

$$
\left\{\begin{array}{rlrlrl}
E_{h, \tau}^{*}(h \underline{m}, 0) & =0 & & \text { for } & h \underline{m} \in \mathbb{R}_{h}^{3} \\
\left(\left(1-i \tau \Delta_{h}\right) E_{h, \tau}^{*}\right)(h \underline{m}, \tau k) & =\delta_{h}(h \underline{m}) & & \text { if } & h \underline{m} \in \mathbb{R}_{h}^{3} \\
\left(\left(1-i \tau \Delta_{h}\right) E_{h, \tau}^{*}\right)(h \underline{m}, \tau(k+1)) & = & E_{h, \tau}^{*}(h \underline{m}, \tau k) & & \text { if } & h \underline{m} \in \mathbb{R}_{h}^{3}, \tau k \in \mathbb{R}_{0}^{+}, k \in \mathbb{Z}^{+}
\end{array}\right.
$$

We note that it is also possible to describe the fundamental solution by application of $R_{h} F$

$$
E_{h, \tau}^{*}(h \underline{m}, \tau k)=R_{h} \mathcal{F}\left(\frac{H(t)}{2 \pi}\left(1+i \tau d^{2}\right)^{-\frac{t}{\tau}} \chi_{h}(\xi)\right)(h \underline{m}, \tau k) .
$$

However, this does not proves our assertion, that of $E_{h, \tau}^{*}$ being a fundamental solution of (2). For that purpose, we need to do a similar study as in Section 2.2 .

\subsection{Existence of Fundamental Solution}

First we have the following three lemmas

Lemma 4.1. Let $f_{h}$ be an arbitrary bounded function. Then the equation

$$
\left(1-i \tau \Delta_{h}\right) v_{h}=f_{h}(x),
$$

for all $x \in \mathbb{R}_{h}^{3}$ has a unique solution $v_{h}$.

Proof. We will omit the presentation of this proof because it is very similar to the proof of the Lemma 1 presented in [5].

The following two lemmas are proved in [5]. 
Lemma 4.2. If $f_{h} \in l_{1}\left(\mathbb{R}_{h}^{3}\right)$, then $v_{h} \in l_{1}\left(\mathbb{R}_{h}^{3}\right)$.

Lemma 4.3. If $\left|f_{h}(x)\right|<K_{1} e^{-c_{1}|x|}$ and $\left|e_{h}(x)\right| \leq K_{2} e^{-c_{2}|x|}$ with $0<c_{1}<c_{2}$, then $v_{h}(x) \leq K_{5} e^{-\left(c_{1}-\delta\right)|x|}$ for all $\delta>0$.

With this three results we can present the following result about the existence of the fundamental solution.

Theorem 4.4. System (19) has a unique solution $E_{h, \tau}^{*}$ and for arbitrary $T_{0}<$ $\infty$ it holds $E_{h, \tau}^{*} \in l_{1}\left(\mathbb{R}_{h}^{3} \times\left[0, T_{0}\right]\right)$.

Proof. The assertion follows from Lemmas 4.14 .2 and 4.3. We remark that the consideration in Lemma 4.3 can be repeated as long as necessary. An estimation of the $l_{1}$-norm with respect to $t$ is possible because the number of time steps is bounded.

\subsection{Convergence}

For $t=0$ we can write the difference equation in the form

$$
\begin{aligned}
\left(-i+\frac{6 \tau}{h^{2}}\right) & E_{h, \tau}^{*}(h \underline{m}, \tau k)=\delta_{h}(h \underline{m}) \\
& +\frac{\tau}{h^{2}}\left[E_{h, \tau}^{*}\left(h\left(m_{1}+1\right), h m_{2}, h m_{3}, \tau k\right)+E_{h, \tau}^{*}\left(h\left(m_{1}-1\right), h m_{2}, h m_{3}, \tau k\right)\right. \\
& +E_{h, \tau}^{*}\left(h m_{1}, h\left(m_{2}+1\right), h m_{3}, \tau k\right)+E_{h, \tau}^{*}\left(h m_{1}, h\left(m_{2}-1\right), h m_{3}, \tau k\right) \\
& \left.+E_{h, \tau}^{*}\left(h m_{1}, h m_{2}, h\left(m_{3}+1\right), \tau k\right)+E_{h, \tau}^{*}\left(h m_{1}, h m_{2}, h\left(m_{3}-1\right), \tau k\right)\right],
\end{aligned}
$$

We have that

$$
\left|-i+\frac{6 \tau}{h^{2}}\right| \sum_{\underline{m} \in \mathbb{R}_{h}^{3}}\left|E_{h, \tau}^{*}(h \underline{\underline{m}}, \tau)\right| h^{3} \leq 1+\frac{6 \tau}{h^{2}} \sum_{\underline{m} \in \mathbb{R}_{h}^{3}}\left|E_{h, \tau}^{*}(h \underline{m}, \tau)\right| h^{3},
$$

which implies that

$$
\left\|E_{h, \tau}^{*}(\cdot, \tau)\right\|_{l_{1}\left(\mathbb{R}_{h}^{3}\right)} \leq 1 .
$$

In the same way we prove that the inequality

$$
\left\|E_{h, \tau}^{*}(\cdot, \tau(k+1))\right\|_{l_{1}\left(\mathbb{R}_{h}^{3}\right)} \leq\left\|E_{h, \tau}^{*}(\cdot, \tau k)\right\|_{l_{1}\left(\mathbb{R}_{h}^{3}\right)},
$$

for each $k \geq 1$ starting with the corresponding equations (19). We obtain

$$
\begin{aligned}
\left\|E_{h, \tau}^{*}\right\|_{\left.\left.l_{1}\left(G_{h} \times\right] 0, T_{0}\right]_{\tau}\right)} & \leq\left\|E_{h, \tau}^{*}\right\|_{\left.\left.l_{1}\left(\mathbb{R}_{h}^{3} \times\right] 0, T_{0}\right]_{\tau}\right)} \\
& \leq \sum_{k=1}^{m_{0}} \tau=T_{0} .
\end{aligned}
$$


In relation to the continuous fundamental solution we have the result (8)

$$
\begin{aligned}
\left\|R_{\tau} R_{h} E\right\|_{\left.\left.l_{1}\left(G_{h} \times\right] 0, T_{0}\right]_{\tau}\right)} & =\sum_{k=1}^{m_{0}} \frac{\operatorname{Vol}\left(G_{h}\right)}{(4 \pi k \tau)^{\frac{3}{2}}} \tau \\
& =\frac{\operatorname{Vol}\left(G_{h}\right)}{(4 \pi \tau)^{\frac{3}{2}} \tau^{\frac{1}{2}}} \sum_{k=1}^{m_{0}} \frac{1}{k^{\frac{3}{2}}} \\
& \leq\left\{\begin{array}{ccc}
\operatorname{Vol}\left(G_{h}\right) \frac{T_{0}}{(4 \pi)^{\frac{3}{2}}} & \text { if } & \tau \geq 1 \\
\operatorname{Vol}\left(G_{h}\right) \frac{T_{0}+m_{0}^{2}}{(4 \pi)^{\frac{3}{2}}} & \text { if } & 0<\tau<1
\end{array} .\right.
\end{aligned}
$$

Now we study

$$
\left\|E_{h, \tau}^{*}(\cdot, \tau k)-R_{h} E(\cdot, \tau k)\right\|_{l_{1}\left(G_{h}\right)},
$$

para $k>m_{0}$. In order to estimate the right hand side of (10) we need to study the following inequality, which is very similar to (12)

$$
\begin{gathered}
\left\|\left(\mathcal{F}_{h} E_{h, \tau}^{*}\right)(\cdot, \tau k)-\left(R_{\tau} R_{h} \mathcal{F}^{-1} E^{\epsilon}\right)(\cdot, \tau k)\right\|_{L_{1}\left(\mathbb{R}^{3}\right)} \\
\leq \frac{H(\tau k)}{(2 \pi)^{\frac{3}{2}}}\left(\frac{2 \tau k}{\epsilon}\right)^{\frac{3}{2}} \\
\cdot \underbrace{}_{(V)} \underbrace{\left\|\left(\frac{\epsilon}{2 \tau k}\right)^{\frac{3}{2}}\left(1+i \tau d^{2}\right)^{-k}-\exp \left(-\frac{\tau k d^{2}}{\epsilon}\right) \exp \left(-i\left(\tau k d^{2}-\frac{3 \pi}{4}\right)\right)\right\|_{L_{1}\left(\mathbb{R}^{3}\right)}}_{-\exp \left(-\frac{\tau k|\xi|^{2}}{\epsilon}\right)^{\frac{3}{2}} \exp \left(-i\left(\tau k|\xi|^{2}-\frac{3 \pi}{4}\right)\right) \|_{L_{1}\left(\mathbb{R}^{3}\right)}} \\
+\| \exp \left(-\frac{\tau k d^{2}}{\epsilon}\right)^{\frac{3}{2}} \exp \left(-i\left(\tau k d^{2}-\frac{3 \pi}{4}\right)\right)
\end{gathered}
$$

Taking into account that $(V I)=(I V)$ and the following relation

$$
\begin{aligned}
& \left|\left(\frac{\epsilon}{2 \tau k}\right)^{\frac{3}{2}}\left(1+i \tau d^{2}\right)^{-k}-\exp \left(-\frac{\tau k d^{2}}{\epsilon}\right) \exp \left(-i\left(\tau k d^{2}-\frac{3 \pi}{4}\right)\right)\right| \\
& \quad \leq\left(\frac{\epsilon}{2 \tau k}\right)^{\frac{3}{2}}\left(1+\tau^{2}\left(h^{2}-1\right)^{2}|\xi|^{4}\right)^{k}+\exp \left(-\frac{\tau k\left(h^{2}-1\right)|\xi|^{2}}{\epsilon}\right),
\end{aligned}
$$


we conclude that the results obtained are equal to the conclusions obtained in (17). In this sense we can say that

$$
\left\|E_{h, \tau}^{*}-R_{\tau} R_{h} E\right\|_{l_{1}\left(G_{h} \times\left(T_{0},+\infty\right)_{\tau}\right)} \leq C_{2}(\epsilon, h, \tau)
$$

where $C_{2}(\epsilon, h, \tau)$ is a quantity that tends to zero when $\epsilon<\tau^{8}$ and $\frac{\tau}{h^{2}}<\frac{1}{6 \pi^{2}}$. From (20) and (22) we obtain

$$
\leq\left\{\begin{array}{ccc}
\left\|E_{h, \tau}^{*}-R_{\tau} R_{h} E\right\|_{l_{1}\left(G_{h} \times\left(T_{0},+\infty\right)\right)} \\
T_{0}+\operatorname{Vol}\left(G_{h}\right) \frac{T_{0}}{(4 \pi)^{\frac{3}{2}}}+C_{2}(h, h, \tau) \quad \text { if } & \tau \geq 1 \wedge \epsilon<\tau^{8} \wedge \frac{\tau}{h^{2}}<\frac{1}{6 \pi^{2}} \\
T_{0}+\operatorname{Vol}\left(G_{h}\right) \frac{T_{0}+m_{0}^{2}}{(4 \pi)^{\frac{3}{2}}}+C_{2}(h, h, \tau) & \text { if } & 0<\tau<1 \wedge \epsilon<\tau^{8} \wedge \frac{\tau}{h^{2}}<\frac{1}{6 \pi^{2}}
\end{array} .\right.
$$

In this conditions we can present the following theorem

Theorem 4.5. For $h \rightarrow 0$ and $\tau \rightarrow 0$ we have the convergence

$$
\left\|E_{h, \tau}^{*}(\cdot, t)-R_{\tau} R_{h} E(\cdot, t)\right\|_{l_{1}\left(G_{h} \times \mathbb{R}_{\tau}^{+}\right)} \rightarrow 0 .
$$

Proof. For an arbitrary $\delta$ we can choose $h$ and $\tau$ such that

$$
\left\|E_{h, \tau}^{*}(\cdot, t)-R_{\tau} R_{h} E(\cdot, t)\right\|_{l_{1}\left(G_{h} \times \mathbb{R}_{\tau}^{+}\right)} \leq \delta .
$$

If we consider the same $T_{0}$ of the Theorem 1 and we use $T_{0}^{+}$in (20) and $T_{0}^{-}$for (22) we obtain the desired result.

Acknowledgement The second author wishes to express his gratitude to Fundação para a Ciência e a Tecnologia for the support of his work via the grant SFRH/BSAB/495/2005.

\section{References}

[1] P. Cerejeiras, U. Kähler and F. Sommen, "Parabolic Dirac Operators and the Navier-Stokes equations over time-varying domains", Math. Meth. in Appli. Sc. 28, 2005, pps 1715-1724.

[2] P. Cerejeiras and N. Vieira, "Factorization of the non-linear Schrödinger Operator", Advances in Applied Clifford Algebras 17, 2007.

[3] C. R. Deeter and G. Springer, "Discrete harmonic kernels", J. Math. Mech. 14, 1965, pps 413-438.

[4] R. Delanghe, F. Sommen and V. Souček, "Clifford algebras and spinor-valued functions", Kluwer Academic Publishers, Dordrecht, 1992.

[5] K. Gürlebeck and A. Hommel, "On finite difference Dirac operators and their fundamental solutions", Adv. Appl. Clifford Algebras 11, 2001, pps 89- 106. 
[6] K. Gürlebeck and A. Hommel, "On Fundamental Solutions of the Heat Conduction Difference Operator", Journal for Analysis and its Applications 13, 1994, pps 1-16.

[7] K. Gürlebeck and W. Sprößig, "Quaternionic and Clifford calculus for Engineers and Physicists", John Wiley \&. Sons, Chichester, 1997.

[8] F. Stummel, "Elliptische Differenzenoperatoren unter Dirichletrandbedingungen", Math. Z. 97, 1967, pps 169-211.

[9] G. Velo, "Mathematical Aspects of the nonlinear Schrödinger Eqauation", Vázquez, Luis et al. (ed.), Proceedings of the Euroconference on nonlinear KleinGordon and Schrödinger systems: theory and applications, Singapore: World Scientific. 39-67 (1996). 